\title{
Maximum work from a finite reservolr by sequential Carnot cycles
}

\author{
Mary Jo Ondrechen, Bjarne Andresen,a) Michael Mozurkewich, and \\ R. Stephen Berry \\ Department of Chemistry and the James Franck Institute, The University of Chicago, Chicago, Illinois 60637
}

\begin{abstract}
The production of work from a heat source with finite heat capacity is discussed. We examine the conversion of heat from such a source first by a single Carnot engine and then by a sequence of Carnot engines. The optimum values of the operating temperatures of these engines are calculated. The work production and efficiency of a sequence with an arbitrary number of engines is derived, and it is shown that the maximum available work can be extracted only when the number of cycles in the sequence becomes infinite. The results illustrate the importance of recovery or bottoming processes in the optimization of work-producing systems. In addition, the present model illuminates one practical limitation of the Carnot cycle: The Carnot efficiency is only obtainable from a heat source with infinite heat capacity. However, another cycle, somewhat reminiscent of the Otto and Brayton cycles, is derived which will provide the maximum efficiency for a heat source with a finite heat capacity.
\end{abstract}

\section{INTRODUCTION}

It is well known that Carnot engines have little practical use because of the large volumes they sweep out on the adiabatic branches. This limitation takes expression in a low value of the work ratio, the net work per cycle divided by the work done in the expansion stroke. For the Carnot engine, the expansion stroke is the hot isotherm plus the adiabatic expansion. Recall that the work done on the ideal Carnot engine in adiabatic compression equals the work done by the engine in adiabatic expansion. Moreover this work ratio is sensitive to irreversibilities, especially to mechanical friction. 1.2 Investigations of the optimum performance and irreversibilities of engines operating in finite time have only very recently been reported..$^{3-6}$ While the Carnot cycle (i.e., a cycle consisting of two adiabats and two isotherms) delivers the maximum work in an infinitely slow cycle when irreversibilities are present, it is not the optimum cycle for processes operating in finite time..$^{5,6}$

However, even if a Carnot engine is operated reversibly, the Carnot efficiency

$$
\eta_{\text {Carnot }}=\left(T^{\text {high }}-T^{\text {low }}\right) / T^{\text {high }}
$$

cannot be realized if the heat which drives the engine is supplied by a source with finite heat capacity. As heat is withdrawn from the source, its temperature drops and the available work decreases.

Consider a Carnot engine operating between two reservoirs with temperatures $T^{H}$ and $T^{L}$. Suppose that the source with constant heat capacity $C$ is initially at temperature $T^{S}$, with $T^{S}>T^{H}>T^{L}$. Suppose next that the source transfers its heat to the reservoir at $T^{H}$, while its temperature drops from $T^{S}$ to $T^{H}$, and the source and reservoir are then disconnected. The heat transferred to the engine is $C\left(T^{S}-T^{H}\right)$. This heat may then be converted into work with efficiency $\left(T^{H}-T^{L}\right) / T^{H}$. If $T^{H}$ is set high (i.e., close to $T^{S}$ ) the heat transferred to the engine is small, but it will be converted into work with high efficiency. Conversely, if $T^{H}$ is set low (i.e., close to $T^{L}$ ), a larger amount of heat is transferred to the engine, but it is converted into work with low efficiency.
In this paper we investigate a system with heat supplied by a source with finite, constant heat capacity and in which this heat is converted into work in two different ways: in the first case, by a single Carnot engine; and in the second case, by a sequence of Carnot cycles. The optimum value for $T^{H}$. the upper temperature of each engine, is derived both for the single engine case and for a sequence of $N$ Carnot cycles. The work produced by an arbitrary number of optimized engines is calculated, and it is shown that the maximum work is extracted (i.e., the effectiveness of the system becomes unity) only by an infinite number of Carnot cycles $(N \rightarrow \infty)$. From our expression for the work in the limit $N$ $\rightarrow \infty$, it is apparent that for a finite heat source originally at $T^{S}$ and a cold reservoir at $T^{L}$, the Carnot efficiency $\left(T^{S}\right.$ $\left.-T^{L}\right) / T^{S}$ is unobtainable, even by an infinite number of sequential Carnot engines. Finally, the reversible cycle which achieves the maximum efficiency from a finite hot reservoir is constructed, and some practical consequences of our results are discussed.

\section{ONE-ENGINE OPTIMIZATION}

For the case where work is produced by a single Carnot engine, the work is given by

$$
\begin{gathered}
W=C\left(T^{S}-T^{H}\right)\left(T^{H}-T^{L}\right) / T^{H}, \\
W=C\left[T^{S}+T^{L}-T^{H}-\left(T^{S} T^{L} / T^{H}\right)\right] .
\end{gathered}
$$

The temperatures $T^{S}$ and $T^{L}$, which might be, for example, the flame temperature of a combustion process and ambient temperature, respectively, are regarded as fixed and $T^{H}$ is the variable parameter. The upper temperature $T^{H}$ for which the work is maximized is the solution to

$$
\frac{d W}{d T^{H}}=C\left[T^{S} T^{L} /\left(T^{H}\right)^{2}-1\right]=0 .
$$

The optimum value for $T^{H}$ is then

$$
T^{H}(1)=\left(T^{S} T^{L}\right)^{1 / 2}
$$

the script letters denote the optimum value, and 1 in parentheses denotes one Carnot cycle. 


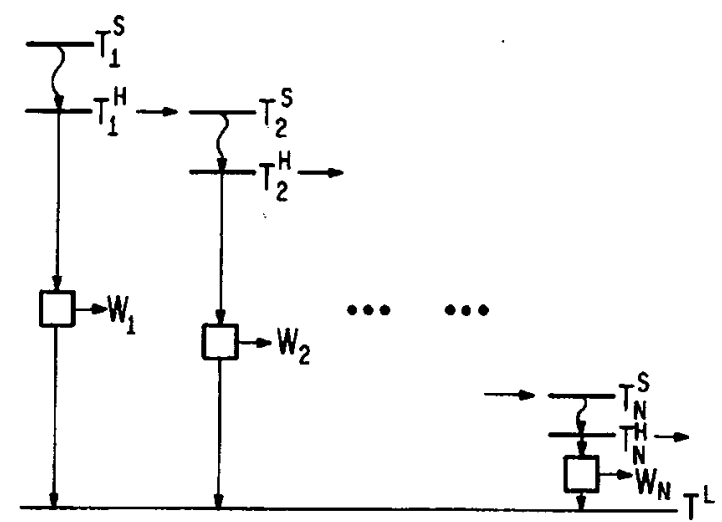

Fig. 1. Sequence of $N$ Carnot cycles. Heat is supplied to each engine at $T_{i}^{H}$ from a source initially at $T_{i}^{S}$. The source is disconnected from the $i$ th engine at $T_{i}^{H}$ and then becomes the source for the $(i+1)$ th engine.

\section{N-ENGINE OPTIMIZATION}

In the one-engine case, the heat source is disconnected from the hot reservoir of the system at $T^{H}$. This means that a significant amount of the original availability is not recovered, because $C\left(T^{H}-T^{L}\right)$ units of heat are discarded unless extraneous processes can utilize this lower-grade heat. Naturally, the effectiveness can be improved if engines are added to the system (see Fig. 1). The high temperature $T_{\uparrow}^{H}$ of the first cycle becomes the source temperature of the second cycle $T_{2}^{S}$ and so on so that

$$
T_{i \cdot}^{H}=T_{i+1}^{S}
$$

for $1 \leq i \leq(N-1)$, where the subscripts denote the element of the sequence and where $N$ is the total number of cycles in the sequence. ${ }^{7}$

The work produced by the $i$ th cycle in the sequence is [cf. Eq. (3)]

$$
W_{i}=C\left[T_{i}^{S}+T^{L}-T_{i}^{H}-\left(T_{i}^{S} T^{L} / T_{i}^{H}\right)\right] .
$$

The total work for the $N$-cycle sequence is

$$
W(N)=\sum_{i=1}^{N} W_{i}
$$

which, using Eqs. (6) and (7), becomes

$$
W(N)=C \sum_{i=1}^{N}\left(-T_{i}^{S} T^{L} / T_{i}^{H}\right)+C T^{L} N+C T_{1}^{S}-C T_{N}^{H}
$$

We wish to find the values for the elements of the set $\left\{T_{1}^{H}\right.$, $\left.T_{2}^{H}, \ldots, T_{N}^{H}\right\}$ for which $W(N)$ is maximized. This is, in effect, a problem in discrete optimal control, albeit exceedingly simple, since the only variable in the $N$-cycle problem is $T_{1}^{H}$. This is because $T_{N}^{H}$ may be fixed as the optimum $T^{H}$ for the one-cycle problem, ..., and $T_{2}^{H}$ may be fixed by optimization of the $(N-1)$-cycle problem. In other words, any $T_{i}^{H}$ may be optimized independently of any engines with index less than $i$.

Hence, for the two-cycle case,

$$
\begin{aligned}
W(2)=-C\left(T_{1}^{S} T^{L} / T_{1}^{H}\right) & -C\left(T_{1}^{H} T^{L} / T_{2}^{H}\right) \\
& +2 C T^{L}+C T_{1}^{S}-C T_{2}^{H},
\end{aligned}
$$

but the optimum value for $T_{2}^{H}$ is given by

$$
T_{2}^{H}(2)=\sqrt{T_{2}^{S} T^{L}}=\sqrt{T_{1}^{H} T^{L}} .
$$

If $T_{2}^{H}$, the high temperature of the second cycle of the two-cycle sequence, is fixed at this optimum value, then $\mathcal{T}_{\mathrm{I}}^{H}(2)$ is the solution to

$$
\frac{d W(2)}{d T_{1}^{H}}=C\left[T_{1}^{S} T^{L} /\left(T_{1}^{H}\right)^{2}-\left(T_{1}^{H}\right)^{-1 / 2}\left(T^{L}\right)^{1 / 2}\right]=0
$$

given by

$$
T_{1}^{H}(2)=\left[\left(T_{1}^{S}\right)^{2} T^{L}\right]^{1 / 3}=T_{1}^{S}\left[T^{L} / T_{1}^{S}\right]^{1 / 3} .
$$

It is straightforward to prove that, for an $N$-cycle sequence with the high temperature of each cycle optimized, the optimum value for $T_{1}^{H}(N)$, the upper temperature of the first engine in the sequence, is

$$
\mathcal{T}_{1}^{H}(N)=\left[\left(T_{1}^{S}\right)^{N} T^{L}\right]^{1 / N+1}=T_{1}^{S}\left[T^{L} / T_{1}^{S}\right]^{1 /(N+1)} .
$$

Equation (14) has already been shown to be true for $N$ $=1$ and 2; we prove it by induction for an arbitrary $N$. It is easiest to prove the case for $N$ engines from the case for $N$ -1 if we count backwards. First, we suppose that Eq. (14) gives the optimum upper temperature of engine 1 for any sequence if the total number of cycles in the sequence is any integer between 1 and $N-1$ inclusive, and if all engines in the sequence with index $\geq 2$ have already been optimized. We then must show that Eq. (14) holds for a sequence of $N$ cycles (provided, of course, that the upper temperatures of engines 2 through $N$ in this sequence have been set at their optimum values). Since each engine may be optimized independently of engines of lower index, we start with engine $N$ in the $N$-cycle sequence (see Fig. 1) and then proceed to engines $N-1, N-2, \ldots, 2$ (i.e., we work from right to left in Fig. 1). We have assumed that Eq. (14) is true for any sequence with 1 through $N-1$ cycles; this gives the optimum upper temperature for $2 \leq i \leq N$ in the $N$ engine problem:

$$
T_{i}^{H}(N)=T_{i}^{S}\left(T^{L} / T_{i}^{S}\right)^{1 /(N-i+2)} .
$$

We now wish to recast Eq. (15) in terms of $T_{1}^{H}$, the upper temperature of the first engine. To do this we use Eq. (15) with $i=2$ and substitute Eq. (6) to find the optimized upper temperature of the second engine:

$$
T_{2}^{H}(N)=T_{1}^{H}\left(T^{L} / T_{1}^{H}\right)^{1 / N} .
$$

We also need to express $\mathcal{T}_{i}^{H}(N)$ for $3 \leq i \leq N$ in terms of $T_{1}^{H}$; hence, we must prove Eq. (18) below. Equation (18) has already been shown to be true for $i=2$ [see Eq. (16)]; we prove it for $3 \leq i \leq N$ by induction on $i$. It is assumed that, for the $(i-1)$ th engine,

$$
\tau_{i-1}^{H}(N)=T_{!}^{H}\left(T^{L} / T_{\uparrow}^{H}\right)^{(i-2) / N}=T_{i}^{S} .
$$

We substitute Eq. (17) into Eq. (15) to express the optimized upper temperature of the $i$ th engine in the sequence as a function of $T_{\mathrm{l}}^{H}$ :

$$
\tau_{i}^{H}(N)=T_{1}^{H}\left(T^{L} / T_{1}^{H}\right)^{(i-1) / N} .
$$

Finally, substitution of Eq. (18) into the expression for the work produced by the $N$-cycle sequence, Eq. (9) yields

$$
\begin{array}{r}
W(N)=C\left[-T_{1}^{S} T^{L} / T_{1}^{H}-N\left(T_{1}^{H}\right)^{1 / N}\left(T^{L}\right)^{(N-1) / N}\right. \\
\left.+T^{L} N+T_{1}^{S}\right] .
\end{array}
$$

Again, setting the first derivative of $W$ with respect to $T_{\perp}^{H}$ equal to zero, one obtains 


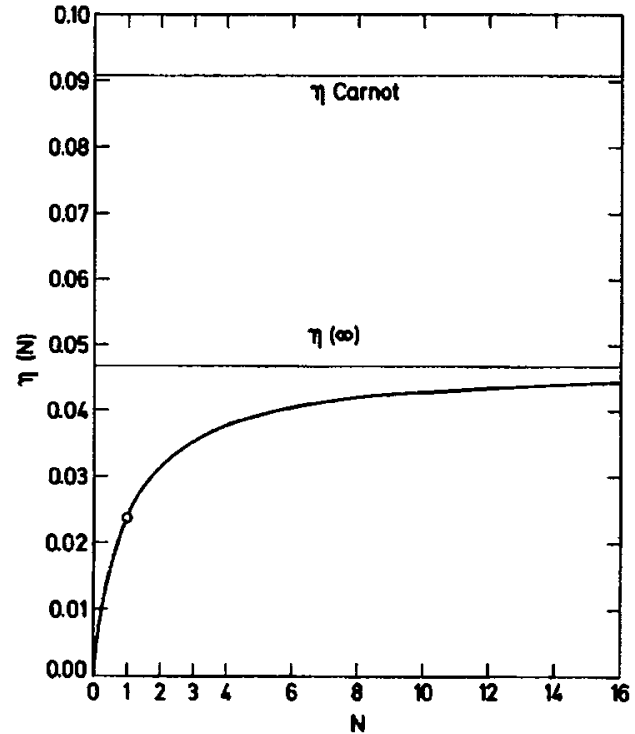

Fig. 2. Thermal efficiency of optimized $N$ sequence of Carnot engines for a range of $N$. Also shown are the reversible efficiencies of conversion from a finite capacity heat source, $\eta(\infty)$, and from an infinite capacity heat source, $\eta$ carnor. The initial ratio of reservoir temperatures is $T_{1}^{S} / T^{L}$ $=1.1$.

$$
C\left\{T_{1}^{S} T^{L} /\left[\mathcal{T}_{1}^{H}(N)\right]^{2}-\left[T^{L} / \mathcal{T}_{1}^{H}(N)\right]^{(N-1) / N}\right\}=0,
$$

from which Eq. (14) follows.

Hence, if each cycle in the $N$-member sequence has been optimized [i.e., the upper temperature has been fixed according to Eq. (15)], the maximum work obtainable by this sequence from a source with constant heat capacity initially at $T_{1}^{S}$ is

$$
W(N)=C\left[-(N+1) T^{L}\left(T_{1}^{S} / T^{L}\right)^{1 /(N+1)}+N T^{L}+T_{1}^{S}\right] .
$$

The upper limit of this function is achieved for $N \rightarrow \infty$. We rewrite Eq. (21) as

$$
\begin{aligned}
W(N)=C\left\{T_{1}^{S}-T^{L}-\right. & T^{L}(N+1) \\
& \left.\times\left[\left(T_{1}^{S} / T^{L}\right)^{1 /(N+1)}-1\right]\right\}
\end{aligned}
$$

and use that

$$
\lim _{N \rightarrow \infty} N\left(x^{1 / N}-1\right)=\ln x
$$

to obtain

$$
W(\infty)=C\left[T_{1}^{S}-T^{L}-T^{L} \ln \left(T_{1}^{S} / T^{L}\right)\right],
$$

which is the maximum (reversible) work extractable from the finite capacity heat reservoir, since it is equal to the availability of the original material at $T_{1}^{S}$ (Ref. 8):

$$
W=C \int_{T^{L}}^{T_{1}^{S}}\left(1-\frac{T^{L}}{T}\right) d T \text {. }
$$

This quantity is considerably larger than the maximum work extractable with a single Carnot cycle [combine Eqs. (3) and (5) or use Eq. (21) directly]:

$$
W(1)=C\left(\sqrt{T_{1}^{S}}-\sqrt{T^{L}}\right)^{2} .
$$

The corresponding efficiency of the sequence, $W(N) / Q$ (from source), is

$$
\eta(N)=W(N) /\left[C\left(T_{1}^{S}-T^{L}\right)\right],
$$

which is plotted versus $N$ in Fig. 2 for $T_{1}^{S} / T^{L}=1$.1. Clearly $\eta(N)$ and $W(N)$ have the same functional dependence upon $N$, they are both increasing functions. In the limit $N \rightarrow \infty$ we have

$$
\begin{aligned}
\eta(\infty) & =1-\frac{T^{L}}{T_{1}^{S}-T^{L}} \ln \left(T_{1}^{S} / T^{L}\right) \\
& =1+\frac{1-\eta_{c}}{\eta_{c}} \ln \left(1-\eta_{c}\right)
\end{aligned}
$$

where

$$
\eta_{c} \equiv \eta_{\text {Carnot }}=\left(T_{1}^{S}-T^{L}\right) / T_{1}^{S} .
$$

The values of $\eta(\infty)$ and $\eta_{c}$ are also indicated on Fig. 2 to emphasize the inferiority of $\eta$ (1) (circled). To see more clearly how $\eta(\infty)$ deviates from the Carnot efficiency, we may rewrite Eq. (29) as

$$
\eta(\infty)=\eta_{c}-\left(1-\eta_{c}\right) \sum_{j=1}^{\infty} \frac{\eta_{c}^{j}}{j+1} .
$$

This relationship is depicted in Fig. 3. Since every term in the summation on the right-hand side of Eq. (31) is positive, we have

$$
\eta(\infty)<\eta_{c}
$$

In other words, the efficiency of the infinite sequence of engines producing work from a finite hot reservoir originally at $T_{1}^{S}$ and a cold reservoir at constant temperature $T^{L}$ will always be smaller than the efficiency of a single Carnot engine operating between two infinite reservoirs at $T_{\text {land }}^{S}$ $T^{L}$. Hence the classical Carnot efficiency (derived for systems operating between infinite reservoirs) is not really useful for the establishment of criteria of merit for heat engines operating with finite heat capacity heat sources. For such engines, the Carnot efficiency is unobtainable.

\section{REVERSIBLE CYCLE}

The reversible limit $N \rightarrow \infty$ was in Eq. (24) derived as an infinite sequence of Carnot cycles. Viewed as a single

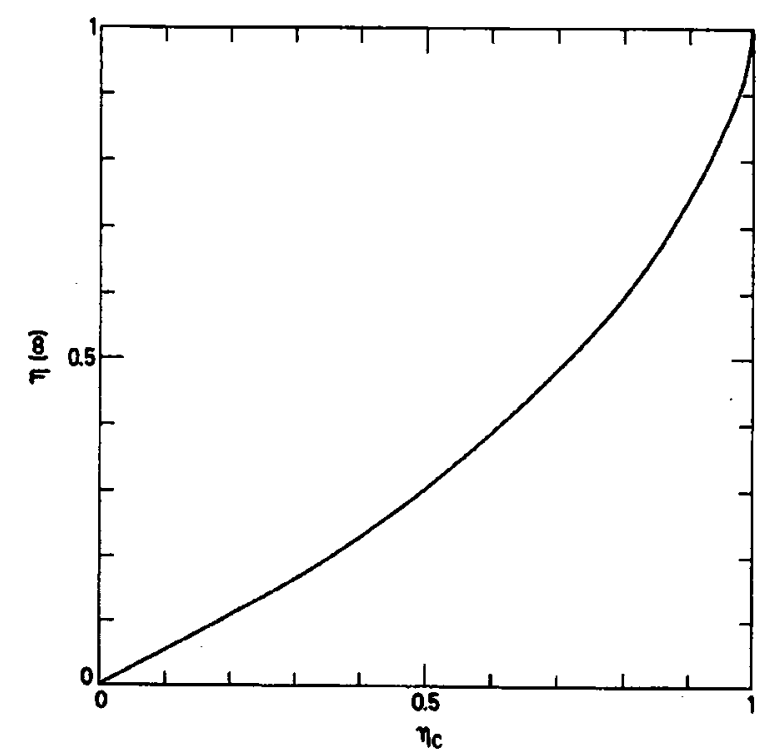

Fig. 3. Thermal efficiency for reversible conversion of energy from a finite capacity heat source versus the efficiency for an infinite capacity heat source (Carnot efficiency) at the same initial temperatures. 
overall cycle, none of the common "named" heat cycles behave this way. We will here construct a cycle (specify its branches) which achieves this reversible energy conversion from a finite capacity heat reservoir.

The low-temperature heat exchange is with an infinite capacity reservoir (at $T^{L}$ ) for which an isothermal branch is appropriate. The high-temperature reservoir has heat capacity $C$, i.e., from the point of view of the engine $-d Q / d T=C$, which, combined with the energy conservation equation for an ideal gas,

$$
d Q=C_{v} d T+(R T / V) d V
$$

yields

$$
\frac{-d V}{V}=\frac{C+C_{v}}{R} \frac{d T}{T}
$$

or in integrated form

$$
V=V_{0}\left(\frac{T_{0}}{T}\right)^{\left(C+C_{r}\right) / R},
$$

where $V_{0}$ and $T_{0}$ are size constants. This defines a polytrope

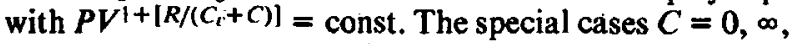
$-C_{v}$, and $-C_{p}$ reproduce the usual adiabat, isotherm, isometric, and isobar, respectively. These two heat exchange branches can now be joined by a reversible adiabat to form the complete reversible finite-heat-capacity reservoir cycle whose $P V$ and TS diagrams are shown in Fig. 4. A finite capacity low-temperature reservoir will of course only change the isotherm into another polytrope with $P V^{1+}\left[R /\left(C^{L}+C_{v}\right)\right]=$ const. The constructed cycle is a hybrid between a Carnot cycle (isothermal part) and something reminiscent of a Brayton or Otto cycle (polytropic part). The exact behavior depends on the relationship between the gas and reservoir heat capacities, $C_{v}$ and $C$, and on the constraints on the reservoir that define what kind of heat capacity $C$ represents, e.g., constant volume, constant pressure, constant surface tension, etc.

Let us close with a remark regarding the comparison of a single large engine with a set of engines of the type described here. The polycycle is, in effect, a series of cycles, each using the waste heat from the one before. Cycles using waste heat are generally called bottoming cycles. The advantage of the polycycle comes from its ability to convert heat to work without requiring the extremely large volumes and compression ratios that a single engine would call for. The work produced by the polycycle relative to the total energy flow (its work ratio) and the compression ratio are necessarily less than the corresponding quantities for the Carnot cycle, because some heat is accepted at less than the maximum temperature. In particular the work efficiency in terms of the swept volume depends strongly upon the initial temperature gap available and the reservoir heat capacity. The largest "volume efficiency," the work divided by $\left(V_{\max }-V_{\min }\right)$, is achieved for

$$
\frac{R}{C+C_{v}}=1+\ln \left(\frac{T_{1}^{S}}{T^{L}}\right) /\left(1-\frac{T_{1}^{S}}{T^{L}}\right)
$$

which makes the polycycle particularly appropriate for bottoming cycles and other conversion of large quantities of low-grade heat into work. For large temperature ratios $\left(T_{1}^{S} / T^{L}\right)$ the rapidly increasing compression ratio can make the cycle extremely sensitive to friction losses from the piston.

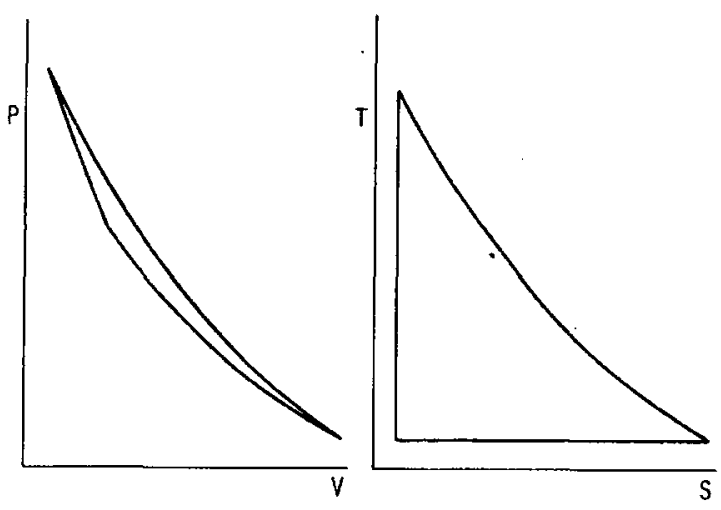

Fig. 4. $P V$ and $T S$ diagrams for the ideal gas reversible polycycle which extracts the maximum possible work from a finite-heat-capacity reservoir. The left branch is the adiabat, the bottom one the isotherm, and the upper-right branch the polytrope. Coordinate scales are arbitrary.

\section{DISCUSSION}

We have seen that the maximum work extractable from a heat source with finite heat capacity, Eq. (24) is considerably larger than that extractable from the same source by a single Carnot cycle, Eq. (26). Nevertheless, the efficiency corresponding to Eq. (24) is less than that of a Carnot engine operating between two infinite heat capacity reservoirs at $T_{1}^{S}$ and $T^{L}$. These results have proved to be important in, for example, the determination of the maximum power which can be produced from a combustion process with finite rate. ${ }^{9}$ Indeed, the heat which drives most real engines is supplied by a source with finite heat capacity.

Some common cycles, such as the Otto or the Rankine, exchange heat along paths with changing temperatures. Such cycles have lower Carnot efficiencies than cycles such as the Carnot or Stirling, where heat exchange occurs isothermally. The lower efficiencies of the former are sometimes cited as evidence for their inferiority relative to the latter type. ${ }^{10}$ The above results show that this claim is only justified when infinite heat reservoirs are involved. For finite heat capacity sources it is best to accept the heat in a nonisothermal manner, following the temperature drop of the reservoir, rather than to accept it at some fixed temperature and discard the remaining temperature drop. Thus for this case a new cycle with a high-temperature polytrope matched to the reservoir heat capacity, and not a Carnot cycle, is actually the best.

The optimum value for the upper temperature of a given cycle depends, as one might expect, upon the number of succeeding (higher index) cycles in the sequence. The addition of recovery or bottoming processes permits one to operate optimally by extracting less heat in each cycle; thus each cycle is operated at as high a temperature as possible.

Efforts in the newly established field of finite-time thermodynamics have demonstrated that the efficiencies of real energy conversion processes are limited by finite-time and finite-rate constraints when irreversibilities are present (see, e.g., Refs. 3-6 and 9). The results of the present work show that even in the realm of reversible thermodynamics, the efficiencies of real engines will be limited by the finite heat capacity of the heat source. 


\section{ACKNOWLEDGMENTS}

We would like to thank the Shell Companies Foundation and Exxon, USA for support of this research, and acknowledge a NATO travel grant. We are grateful for the hospitality of the Aspen Center for Physics, where part of this work was performed.

"Present address: Physics Laboratory 2, Universitetsparken 5, Copenhagen 2100, Denmark.

'Joseph H. Keenan, Thermodynamics (MIT, Cambridge, MA, 1970). Chap. XII.
2S. H. Chue, Thermodynamics (Wiley, New York, 1977), Chap. 6. 'B. Andresen, R. S. Berry, A. Nitzan, and P. Salamon, Phys. Rev. A 15, 2086 (1977).

${ }^{4}$ B. Andresen, P. Salamon, and R. S. Berry, J. Chem. Phys. 66, 1571 (1977).

SM. H. Rubin, Phys. Rev. A 19, 1272 (1979).

"M. H. Rubin, Phys. Rev, A 19, 1277 (1979).

${ }^{7}$ Note that other cycles that do not use isothermal branches, e.g., the Rankine cycle, are essentially of this sort, with $N$ large.

"Sec, e.g., H. B. Callen, Thermodynamics (Wiley, New York, 1960), p. 73.

${ }^{9} \mathrm{M}$. J. Ondrechen, R. S. Berry, and B. Andresen, J. Chem. Phys. 72, 5118 (1980).

10For example, Ref. 1, p. 180. 